\title{
Existence of Solutions to a Second Order Coupled System with Nonlinear Coupled Boundary Conditions
}

\author{
Naseer Ahmad Asif ${ }^{*}$, Imran Talib \\ Department of Mathematics, School of Science and Technology, University of Management and Technology, CII Johar Town, Lahore, \\ Pakistan
}

\section{Email address:}

naseerasif@yahoo.com (N. A. Asif), imrantaalib@gmail.com (I. Talib)

\section{To cite this article:}

Naseer Ahmad Asif, Imran Talib. Existence of Solutions to a Second Order Coupled System with Nonlinear Coupled Boundary Conditions. American Journal of Applied Mathematics. Special Issue: Proceedings of the 1st UMT National Conference on Pure and Applied Mathematics (1st UNCPAM 2015). Vol. 3, No. 3-1, 2015, pp. 54-59. doi: 10.11648/j.ajam.s.2015030301.19

\begin{abstract}
We study existence of solution in the presence of upper and lower solutions of some second-order nonlinear coupled ordinary differential system (ODS for short) depending on first order derivatives with nonlinear coupled boundary conditions (CBCs for short). Our method for nonlinear coupled system with nonlinear CBCs is new and it unifies the treatment of many different second order problems. Nagumo condition is used to define bound for the derivative of the solution. Coupled lower and upper solutions, Arzela-Ascoli theorem and Schauder's fixed point theorem play an important role in establishing the arguments.
\end{abstract}

Keywords: Lower and Upper Solutions, Coupled System, Coupled Boundary Conditions, Arzela-Ascoli Theorem, Schauder's Fixed Point Theorem

\section{Introduction}

In this paper, we study existence of solution in the presence of upper and lower solutions of some second-order nonlinear coupled ODS with nonlinear CBCs of the type

$$
\begin{array}{rr}
-u^{\prime \prime}(t)=f\left(t, u(t), v(t), u^{\prime}(t), v^{\prime}(t)\right), & t \in[0,1], \\
-u^{\prime \prime}(t)=g\left(t, u(t), v(t), u^{\prime}(t), v^{\prime}(t)\right), & t \in[0,1], \\
\phi\left(u(0), v(0), u^{\prime}(0), v^{\prime}(0), u^{\prime}(1), v^{\prime}(1)\right)=(0,0), \\
\psi(u(0), v(0))+(u(1), v(1))=(0,0),
\end{array}
$$

where $f, g:[0,1] \times \mathbb{R} \times \mathbb{R} \times \mathbb{R} \times \mathbb{R} \rightarrow \mathbb{R}, \quad \phi: \mathbb{R}^{6} \rightarrow \mathbb{R}^{2} \quad$ and $\psi: \mathbb{R}^{2} \rightarrow \mathbb{R}^{2}$ are continuous functions. A significant motivation factor for the study of the above system has been the applications of the nonlinear differential equations to the areas of mechanics; population dynamics; optimal control; ecology; biotechnology; harvesting; and physics [11, 15, 16]. Moreover, while dealing with nonlinear ODS mostly authors only focus on attention to the differential systems with uncoupled boundary conditions $[1,4,6]$.

But, on the other hand, very few research work is available where the differential systems are coupled not only in the differential systems but also through the boundary conditions
$[2,9,12,14]$. Our considered System (1.1)-(1.2) deals with the latter case.

The other productive aspect of the article is the generalization of the classical concepts that had been discussed in $[3,5,7,8,10,13,17]$. We mean to say if $\phi(j, k, l, m, n, o)=(l-n, m-o)$ and $\psi(j, k)=(-j,-k)$, then (1.2) implies the periodic boundary conditions (BCs for short). Also if $\phi(j, k, l, m, n, o)=(l+n, m+o) \quad$ and $\psi(j, k)=(j, k)$, then (1.2) implies the anti-periodic BCs. In order to obtain a solution satisfying some initial or BCs and lying between a subsolution and supersolution, we need additional conditions. For example, in the periodic case it suffices that

$$
\begin{aligned}
& \alpha_{1}^{\prime}(0) \geq \alpha_{1}^{\prime}(1), \alpha_{2}^{\prime}(0) \geq \alpha_{2}^{\prime}(1), \\
& \alpha_{1}(1)=\alpha_{1}(0), \alpha_{2}(1)=\alpha_{2}(0), \\
& \beta_{1}^{\prime}(0) \leq \beta_{1}^{\prime}(1), \beta_{2}^{\prime}(0) \leq \beta_{2}^{\prime}(1), \\
& \beta_{1}(1)=\beta_{1}(0), \beta_{2}(1)=\beta_{2}(0),
\end{aligned}
$$

and in the anti-periodic case it suffices that 


$$
\begin{aligned}
& \alpha_{1}^{\prime}(0) \geq-\beta_{1}^{\prime}(1), \alpha_{2}^{\prime}(0) \geq-\beta_{2}^{\prime}(1), \\
& -\alpha_{1}(0)=\beta_{1}(1), \quad-\alpha_{2}(0)=\beta_{2}(1), \\
& -\beta_{1}^{\prime}(1) \leq \alpha_{1}^{\prime}(1), \beta_{2}^{\prime}(0) \leq-\alpha_{2}^{\prime}(1), \\
& \alpha_{1}(1)=-\beta_{1}(0), \alpha_{2}(1)=-\beta_{2}(0),
\end{aligned}
$$

so to generalize the classical results (1.3) and (1.4), the concept of coupled lower and upper solutions is defined in Section 2 that allows us to obtain a solution in the sector $\llbracket \alpha_{1}, \beta_{1} \rrbracket \times \llbracket \alpha_{2}, \beta_{2} \rrbracket$. Also (2.1) implies (1.3) and (1.4).

Definition 1.1. We say that a function $\left(\alpha_{1}, \alpha_{2}\right) \in C^{2}[0,1] \times C^{2}[0,1]$ is a sub solution of (1.1) if

$$
\begin{array}{ll}
-\alpha_{1}^{\prime \prime}(t) \leq f\left(t, \alpha_{1}(t), \alpha_{2}(t), \alpha_{1}^{\prime}(t), \alpha_{2}^{\prime}(t)\right), & t \in[0,1], \\
-\alpha_{2}^{\prime \prime}(t) \leq g\left(t, \alpha_{1}(t), \alpha_{2}(t), \alpha_{1}^{\prime}(t), \alpha_{2}^{\prime}(t)\right), & t \in[0,1],
\end{array}
$$

In the same way, a super solution is a function $\left(\beta_{1}, \beta_{2}\right) \in C^{2}[0,1] \times C^{2}[0,1]$, that satisfies the same inequalities in reverse order. For $u, v \in C^{2}[0,1]$, we define the set

$$
\llbracket u, v \rrbracket=\left\{w \in C^{2}[0,1]: u(t) \leq w(t) \leq v(t), t \in[0,1]\right\} .
$$

Definition 1.2. We say that $f$ and $g$ satisfies Nagumo condition relative to the intervals $\left[\alpha_{1}(t), \beta_{1}(t)\right]$ and $\left[\alpha_{2}(t), \beta_{2}(t)\right]$ respectively, if for

$$
\begin{gathered}
r_{1}=\max \left\{\beta_{1}(0)-\alpha_{1}(1)+\beta_{2}(0)-\alpha_{2}(1),\right. \\
\left.\beta_{1}(1)-\alpha_{1}(0)+\beta_{2}(1)-\alpha_{2}(0)\right\},
\end{gathered}
$$

there exists a constant $M$ such that

$$
\begin{array}{r}
M>\max \left\{r_{1}, \sup _{t \in[0,1]}\left|\alpha_{1}^{\prime}(t)\right|, \sup _{t \in[0,1]}\left|\beta_{1}^{\prime}(t)\right|,\right. \\
\left.\sup _{t \in[0,1]}\left|\alpha_{2}^{\prime}(t)\right|, \sup _{t \in[0,1]}\left|\beta_{2}^{\prime}(t)\right|\right\},
\end{array}
$$

and a continuous function $\xi:[0, \infty) \rightarrow(0, \infty)$ such that

$$
\begin{array}{r}
|f(t, u(t), v(t), r, s)| \leq \xi(|r+s|), \alpha_{1}(t) \leq u(t) \leq \beta_{1}(t), \\
\alpha_{2}(t) \leq v(t) \leq \beta_{2}(t), t \in[0,1], r, s \in \mathbb{R}, \\
|g(t, u(t), v(t), r, s)| \leq \xi(|r+s|), \alpha_{1}(t) \leq u(t) \leq \beta_{1}(t), \\
\alpha_{2}(t) \leq v(t) \leq \beta_{2}(t), t \in[0,1], r, s \in \mathbb{R},
\end{array}
$$

and

$$
\int_{r_{1}}^{M} \frac{d y}{\xi(y)}>2
$$

We finish this introduction with a lemma

Lemma 1.3. Let

$L: C^{1}[0,1] \times C^{1}[0,1] \rightarrow C_{0}[0,1] \times C_{0}[0,1] \times \mathbb{R}^{2} \times \mathbb{R}^{2}$ be defined by

$$
\begin{gathered}
{[L(u, v)](t)=\left(u^{\prime}(t)-u^{\prime}(0)-\lambda \int_{0}^{t} u(s) d s, v^{\prime}(t)-v^{\prime}(0)-\lambda \int_{0}^{t} v(s) d s,\right.} \\
(a u(0)+b u(1), c v(0)+d v(1)),(E u(0)+F u(1), G v(0)+H v(1))),
\end{gathered}
$$

Where $\lambda, a, b, c, d, E, F, G$ and $H$ are real constants such and here that

$$
\begin{gathered}
(a d-b c)(E H-F G)\left(e^{-\sqrt{\lambda}}-e^{\sqrt{\lambda}}\right) \neq 0, \\
C_{0}[0,1]=\left\{w \in C^{2}[0,1]: w(0)=0\right\} . \\
{\left[L^{-1}(y, z, \gamma, \delta, \mu, \varsigma)\right]=\left(C_{1} e^{\sqrt{\lambda} t}+C_{2} e^{-\sqrt{\lambda} t}+\frac{1}{2} \int_{0}^{t} e^{\sqrt{\lambda}(t-s)} y(s) d s\right.} \\
\left.-\frac{1}{2} \int_{0}^{t} e^{\sqrt{\lambda}(s-t)} y(s) d s, C_{3} e^{\sqrt{\lambda} t}+C_{4} e^{-\sqrt{\lambda} t}+\frac{1}{2} \int_{0}^{t} e^{\sqrt{\lambda}(t-s)} z(s) d s-\frac{1}{2} \int_{0}^{t} e^{\sqrt{\lambda}(s-t)} z(s) d s\right),
\end{gathered}
$$

Then $L^{-1}$ exists and is continuous and defined by

where

$$
\begin{aligned}
& C_{1}=\frac{1}{(a d-b c)\left(e^{\sqrt{\lambda}}-e^{-\sqrt{\lambda}}\right)}\left(2 \delta\left(a+b e^{-\sqrt{\lambda}}\right)-d\left(a+b e^{-\sqrt{\lambda}}\right) \int_{0}^{1} e^{\sqrt{\lambda}(1-s)} y(s) d s+d\left(a+b e^{-\sqrt{\lambda}}\right) \int_{0}^{1} e^{\sqrt{\lambda}(s-1)} y(s) d s\right. \\
& \left.-2 \gamma\left(c+d e^{-\sqrt{\lambda}}\right)+b\left(c+d e^{-\sqrt{\lambda}}\right) \int_{0}^{1} e^{\sqrt{\lambda}(1-s)} y(s) d s-b\left(c+d e^{-\sqrt{\lambda}}\right) \int_{0}^{1} e^{\sqrt{\lambda}(s-1)} y(s) d s\right), \\
& C_{2}=\frac{1}{(a d-b c)\left(e^{-\sqrt{\lambda}}-e^{\sqrt{\lambda}}\right)}\left(2 \delta\left(a+b e^{\sqrt{\lambda}}\right)-d\left(a+b e^{\sqrt{\lambda}}\right) \int_{0}^{1} e^{\sqrt{\lambda}(1-s)} y(s) d s+d\left(a+b e^{\sqrt{\lambda}}\right) \int_{0}^{1} e^{\sqrt{\lambda}(s-1)} y(s) d s\right. \\
& \left.-2 \gamma\left(c+d e^{\sqrt{\lambda}}\right)+b\left(c+d e^{\sqrt{\lambda}}\right) \int_{0}^{1} e^{\sqrt{\lambda}(1-s)} y(s) d s-b\left(c+d e^{\sqrt{\lambda}}\right) \int_{0}^{1} e^{\sqrt{\lambda}(s-1)} y(s) d s\right),
\end{aligned}
$$




$$
\begin{aligned}
& C_{3}=\frac{1}{(E H-F G)\left(e^{\sqrt{\lambda}}-e^{-\sqrt{\lambda}}\right)}\left(2 \varsigma\left(E+F e^{-\sqrt{\lambda}}\right)-H\left(E+F e^{-\sqrt{\lambda}}\right) \int_{0}^{1} e^{\sqrt{\lambda}(1-s)} z(s) d s+F\left(E+F e^{-\sqrt{\lambda}}\right) \int_{0}^{1} e^{\sqrt{\lambda}(s-1)} z(s) d s\right. \\
& \left.-2 \mu\left(G+H e^{-\sqrt{\lambda}}\right)+F\left(G+H e^{-\sqrt{\lambda}}\right) \int_{0}^{1} e^{\sqrt{\lambda}(1-s)} z(s) d s-F\left(G+H e^{-\sqrt{\lambda}}\right) \int_{0}^{1} e^{\sqrt{\lambda}(s-1)} z(s) d s\right),
\end{aligned}
$$

and

$$
\begin{aligned}
& C_{4}=\frac{1}{(E H-F G)\left(e^{-\sqrt{\lambda}}-e^{\sqrt{\lambda}}\right)}\left(2 \varsigma\left(E+F e^{\sqrt{\lambda}}\right)-H\left(E+F e^{\sqrt{\lambda}}\right) \int_{0}^{1} e^{\sqrt{\lambda}(1-s)} z(s) d s+F\left(E+F e^{\sqrt{\lambda}}\right) \int_{0}^{1} e^{\sqrt{\lambda}(s-1)} z(s) d s\right. \\
& \left.-2 \mu\left(G+H e^{\sqrt{\lambda}}\right)+F\left(G+H e^{\sqrt{\lambda}}\right) \int_{0}^{1} e^{\sqrt{\lambda}(1-s)} z(s) d s-F\left(G+H e^{\sqrt{\lambda}}\right) \int_{0}^{1} e^{\sqrt{\lambda}(s-1)} z(s) d s\right) .
\end{aligned}
$$

\section{Coupled Lower and Upper Solutions}

To cover different possibilities for the nonlinear boundary functions $\phi$ and $\psi$ we introduce the following concept.

Definition 2.1. We say that $\left(\alpha_{1}, \alpha_{2}\right),\left(\beta_{1}, \beta_{2}\right) \in C^{2}[0,1] \times C^{2}[0,1]$ are coupled lower and upper solutions for the problem (1.1) and (1.2) if $\left(\alpha_{1}, \alpha_{2}\right)$ is a sub solution and $\left(\beta_{1}, \beta_{2}\right)$ is asupersolution for the system $(1.1),\left(\alpha_{1}, \alpha_{2}\right) \leq\left(\beta_{1}, \beta_{2}\right)$, and

$$
\begin{gathered}
\phi\left(\beta_{1}(0), \beta_{2}(0), \beta_{1}^{\prime}(0), \beta_{2}^{\prime}(0), \beta_{1}^{\prime}(1), \beta_{2}^{\prime}(1)\right) \leq(0,0) \\
\leq \phi\left(\alpha_{1}(0), \alpha_{2}(0), \alpha_{1}^{\prime}(0), \alpha_{2}^{\prime}(0), \alpha_{1}^{\prime}(1), \alpha_{2}^{\prime}(1)\right), \\
\phi\left(\beta_{1}(0), \beta_{2}(0), \beta_{1}^{\prime}(0), \beta_{2}^{\prime}(0), \alpha_{1}^{\prime}(1), \alpha_{2}^{\prime}(1)\right) \leq(0,0) \\
\leq \phi\left(\alpha_{1}(0), \alpha_{2}(0), \alpha_{1}^{\prime}(0), \alpha_{2}^{\prime}(0), \beta_{1}^{\prime}(1), \beta_{2}^{\prime}(1)\right), \\
\\
\quad\left(\alpha_{1}(1), \alpha_{2}(1)\right)+\psi\left(\beta_{1}(0), \beta_{2}(0)\right)=(0,0), \\
\quad\left(\beta_{1}(1), \beta_{2}(1)\right)+\psi\left(\alpha_{1}(0), \alpha_{2}(0)\right)=(0,0), \\
\quad\left(\alpha_{1}(1), \alpha_{2}(1)\right)+\psi\left(\alpha_{1}(0), \alpha_{2}(0)\right)=(0,0), \\
\quad\left(\beta_{1}(1), \beta_{2}(1)\right)+\psi\left(\beta_{1}(0), \beta_{2}(0)\right)=(0,0) .
\end{gathered}
$$

Theorem 2.2. Assume that $\left(\alpha_{1}, \alpha_{2}\right),\left(\beta_{1}, \beta_{2}\right)$ are coupled lower and upper solutions for the problem (1.1)-(1.2). Also assume that $f$ and $g$ satisfies a Nagumo condition relative to the intervals $\left[\alpha_{1}(t), \beta_{1}(t)\right]$ and $\left[\alpha_{2}(t), \beta_{2}(t)\right]$ respectively. Suppose that $\phi$ is no ndecreasing in the third and fourth arguments. In addition suppose that the function $\psi$ in $\left[\alpha_{1}(0), \beta_{1}(0)\right] \times\left[\alpha_{2}(0), \beta_{2}(0)\right]$ is monotone and the functions

$$
\begin{aligned}
& \phi_{\left(\alpha_{1}, \alpha_{2}\right)}(x, y):=\phi\left(\alpha_{1}(0), \alpha_{2}(0), \alpha_{1}^{\prime}(0), \alpha_{2}^{\prime}(0), x, y\right), \\
& \phi_{\left(\beta_{1}, \beta_{2}\right)}(x, y):=\phi\left(\beta_{1}(0), \beta_{2}(0), \beta_{1}^{\prime}(0), \beta_{2}^{\prime}(0), x, y\right),
\end{aligned}
$$

have got the same kind of monotonocity as $\psi$, then there exists at least one solution $(u, v) \in \llbracket \alpha_{1}, \beta_{1} \rrbracket \times \llbracket \alpha_{2}, \beta_{2} \rrbracket$ of the problem $(1.1)-(1.2)$. Furthermore, $(-M,-M) \leq\left(u^{\prime}(t), v^{\prime}(t)\right) \leq(M, M), t \in[0,1]$.

Proof. Let $\lambda>0$ and consider the modified system

$$
\begin{aligned}
& -u^{\prime \prime}(t)+\lambda u(t)=F^{*}\left(t, u(t), v(t), u^{\prime}(t), v^{\prime}(t)\right), \quad t \in[0,1], \\
& -v^{\prime \prime}(t)+\lambda v(t)=G^{*}\left(t, u(t), v(t), u^{\prime}(t), v^{\prime}(t)\right), \quad t \in[0,1], \\
& \phi^{*}\left(u(0), v(0), u(1), v(1), u^{\prime}(0), v^{\prime}(0)\right)=(u(0), v(0)), \\
& (u(1), v(1))+\psi^{*}(u(0), v(0))=(0,0),
\end{aligned}
$$

with

$$
\begin{aligned}
& F^{*}(t, u(t), v(t), r, s)=f(t, p(t, u(t)), p(t, v(t)), q(r), q(s))+\lambda p(t, u(t)), \\
& G^{*}(t, u(t), v(t), r, s)=g(t, p(t, u(t)), p(t, v(t)), q(r), q(s))+\lambda p(t, v(t)),
\end{aligned}
$$

$$
\begin{aligned}
& \phi^{*}(j, k, l, m, n, o)=p(0,(j, k)+\phi(j, k, l, m, n, o)), \\
& \psi^{*}(j, k)=\psi(p(0,(j, k))), \\
& p(t,(x, y))= \begin{cases}\left(\beta_{1}(t), \beta_{2}(t)\right) & (x, y)\left(\beta_{1}, \beta_{2}\right) \\
(x, y) & \left(\alpha_{1}, \alpha_{2}\right)(x, y)\left(\beta_{1}, \beta_{2}\right) \\
\left(\alpha_{1}(t), \alpha_{2}(t)\right) & (x, y)\left(\alpha_{1}, \alpha_{2}\right)\end{cases}
\end{aligned}
$$

and

$$
q(x, y)= \begin{cases}(M, M) & (x, y)(M, M) \\ (x, y) & (-M,-M)(x, y)(M, M) \\ (-M,-M) & (x, y)(-M,-M) .\end{cases}
$$

Note that if $(u, v) \in \llbracket \alpha_{1}, \beta_{1} \rrbracket \times \llbracket \alpha_{2}, \beta_{2} \rrbracket$ is a solution of (2.2), then $(u, v)$ is a solution of (1.1)-(1.2).

For the sake of simplicity we divide the proof in three steps:

Step 1: We define the mappings

$$
L, N: C^{1}[0,1] \times C^{1}[0,1] \rightarrow C_{0}[0,1] \times C_{0}[0,1] \times \mathbb{R}^{2} \times \mathbb{R}^{2},
$$




$$
[L(u, v)](t)=\left(u^{\prime}(t)-u^{\prime}(0)-\lambda \int_{0}^{t} u(s) d s, v^{\prime}(t)-v^{\prime}(0)-\lambda \int_{0}^{t} v(s) d s,(u(0), v(0)),(u(1), v(1))\right)
$$

and

$$
\begin{aligned}
& {[N(u, v)](t)=\left(\int_{0}^{t} F^{*}\left(s, u(s), v(s), u^{\prime}(s), v^{\prime}(s)\right) d s, \int_{0}^{t} G^{*}\left(s, u(s), v(s), u^{\prime}(s), v^{\prime}(s)\right) d s,\right.} \\
& \left.\phi^{*}\left(u(0), v(0), u(1), v(1), u^{\prime}(1), v^{\prime}(1)\right),-\psi^{*}(u(0), v(0))\right) .
\end{aligned}
$$

Clearly $N$ is continuous and compact by the direct application of Arzela-Ascoli theorem. Also from Lemma 1.3 with $\quad a=1, \quad b=0, \quad c=1, d=0 \quad$ and $E=0, F=1, G=0, H=1, L^{-1}$ exists and is continuous.

On the other hand, solving (2.2) is equivalent to find a fixed point of

$$
L^{-1} N: C^{1}[0,1] \times C^{1}[0,1] \rightarrow C^{1}[0,1] \times C^{1}[0,1] .
$$

Now, Schauder's fixed point theorem guarantees the existence of at least one fixed point sinceis continuous and compact.

Step 2: If $(u, v)$ is a solution of (2.2), then $(u, v) \in \llbracket \alpha_{1}, \beta_{1} \rrbracket \times \llbracket \alpha_{2}, \beta_{2} \rrbracket$. By definition of $\phi^{*}$, we see that $(u(0), v(0)) \in\left[\alpha_{1}(0), \beta_{1}(0)\right] \times\left[\alpha_{2}(0), \beta_{2}(0)\right]$. Thus, if $\psi$ is no ndecreasing, we have by condition $(2.1)$

$$
\left(\alpha_{1}(1), \alpha_{2}(1)\right)=-\psi\left(\beta_{1}(0), \beta_{2}(0)\right)-\psi(u(0), v(0))=(u(1), v(1))-\psi\left(\alpha_{1}(0), \alpha_{2}(0)\right)\left(\beta_{1}(0), \beta_{2}(0)\right) .
$$

Similarly, if $\psi$ is no nincreasing, then (2.3) holds. Hence $(u(1), v(1)) \in\left[\alpha_{1}(1), \beta_{1}(1)\right] \times\left[\alpha_{2}(1), \beta_{2}(1)\right]$. Now, it remains to show that $(u, v) \in \llbracket \alpha_{1}, \beta_{1} \rrbracket \times \llbracket \alpha_{2}, \beta_{2} \rrbracket$ for $t \in[0,1]$. We claim $(u, v)\left(\beta_{1}, \beta_{2}\right)$. If $(u, v)\left(\beta_{1}, \beta_{2}\right)$, then either $u \quad \beta_{1}$ and/or $v \beta_{2}$. If $u \quad \beta_{1}$, then there exist some $t_{0} \in[0,1]$ such that $u\left(t_{0}\right)-\beta_{1}\left(t_{0}\right)>0$. So, $u-\beta_{1}$ attains apositive maximum at $t_{0} \in[0,1]$. Thus $\left(u-\beta_{1}\right)^{\prime}\left(t_{0}\right)=0$ and $\left(u-\beta_{1}\right)^{\prime \prime}\left(t_{0}\right)<0$. But,

$$
\begin{aligned}
& \left(u-\beta_{1}\right)^{\prime \prime}\left(t_{0}\right)>-F^{*}\left(t_{0}, u\left(t_{0}\right), v\left(t_{0}\right), u^{\prime}\left(t_{0}\right), v^{\prime}\left(t_{0}\right)\right)+\lambda u\left(t_{0}\right)+f\left(t_{0}, \beta_{1}\left(t_{0}\right), \beta_{2}\left(t_{0}\right), \beta_{1}^{\prime}\left(t_{0}\right), \beta_{2}^{\prime}\left(t_{0}\right)\right) \\
& =-f\left(t_{0}, \beta_{1}\left(t_{0}\right), \beta_{2}\left(t_{0}\right), u^{\prime}\left(t_{0}\right), v^{\prime}\left(t_{0}\right)\right)-\lambda \beta_{1}\left(t_{0}\right)+\lambda u\left(t_{0}\right)+f\left(t_{0}, \beta_{1}\left(t_{0}\right), \beta_{2}\left(t_{0}\right), \beta_{1}^{\prime}\left(t_{0}\right), \beta_{2}^{\prime}\left(t_{0}\right)\right) \\
& =-f\left(t_{0}, \beta_{1}\left(t_{0}\right), \beta_{2}\left(t_{0}\right), \beta_{1}^{\prime}\left(t_{0}\right), \beta_{2}^{\prime}\left(t_{0}\right)\right)-\lambda \beta_{1}\left(t_{0}\right)+\lambda u\left(t_{0}\right)+f\left(t_{0}, \beta_{1}\left(t_{0}\right), \beta_{2}\left(t_{0}\right), \beta_{1}^{\prime}\left(t_{0}\right), \beta_{2}^{\prime}\left(t_{0}\right)\right) \\
& =\lambda\left(u\left(t_{0}\right)-\beta_{1}\left(t_{0}\right)\right)>0,
\end{aligned}
$$

a contradiction. Similarly one can show that $\left(\alpha_{1}, \alpha_{2}\right) \leq(u, v)$. Hence $(u, v) \in \llbracket \alpha_{1}, \beta_{1} \rrbracket \times \llbracket \alpha_{2}, \beta_{2} \rrbracket$.

Step 3: If $(u, v)$ is a solution of (2.2) then $(u, v)$ satisfies (1.2).

We claim

$$
\begin{gathered}
\left(\alpha_{1}(0), \alpha_{2}(0)\right)(u(0), v(0))+\phi\left(u(0), v(0), u^{\prime}(0), v^{\prime}(0), u^{\prime}(1), v^{\prime}(1)\right) \\
\left(\beta_{1}(0), \beta_{2}(0)\right)
\end{gathered}
$$

If

$$
\left(\alpha_{1}(0), \alpha_{2}(0)\right)(u(0), v(0))+\phi\left(u(0), v(0), u^{\prime}(0), v^{\prime}(0), u^{\prime}(1), v^{\prime}(1)\right)
$$

then

$$
\begin{aligned}
& (u(0), v(0))=\phi^{*}\left(u(0), v(0), u(1), v(1), u^{\prime}(0), v^{\prime}(0)\right) \\
& =p\left(0,(u(0), v(0))+\phi\left(u(0), v(0), u^{\prime}(0), v^{\prime}(0), u^{\prime}(1), v^{\prime}(1)\right)=\left(\alpha_{1}(0), \alpha_{2}(0)\right)\right.
\end{aligned}
$$

Similarly if $\psi$ is no ndecreasing then we have

$$
(u(1), v(1))=-\psi(u(0), v(0))=-\psi\left(\alpha_{1}(0), \alpha_{2}(0)\right)=\left(\beta_{1}(1), \beta_{2}(1)\right) .
$$

Using (2.5), (2.6) and Step 2, we have $\left(u^{\prime}(0), v^{\prime}(0)\right)\left(\alpha_{1}^{\prime}(0), \alpha_{2}^{\prime}(0)\right)$ and $\left(u^{\prime}(1), v^{\prime}(1)\right)\left(\beta_{1}^{\prime}(1), \beta_{2}^{\prime}(1)\right)$. But 


$$
\begin{aligned}
& (u(0), v(0))=\phi\left(u(0), v(0), u(1), v(1), u^{\prime}(0), v^{\prime}(0)\right) \\
& =\left(\alpha_{1}(0), \alpha_{2}(0)\right)+\phi\left(\alpha_{1}(0), \alpha_{2}(0), u^{\prime}(0), v^{\prime}(0), u^{\prime}(1), v^{\prime}(1)\right)\left(\alpha_{1}(0), \alpha_{2}(0)\right)+\phi\left(\alpha_{1}(0), \alpha_{2}(0), \alpha_{1}^{\prime}(0), \alpha_{2}^{\prime}(0), u^{\prime}(1), v^{\prime}(1)\right) \\
& =\left(\alpha_{1}(0), \alpha_{2}(0)\right)+\phi_{\left(\alpha_{1}, \alpha_{2}\right)}\left(u^{\prime}(1), v^{\prime}(1)\right)\left(\alpha_{1}(0), \alpha_{2}(0)\right)+\phi\left(\alpha_{1}(0), \alpha_{2}(0), \alpha_{1}^{\prime}(0), \alpha_{2}^{\prime}(0), \beta_{1}^{\prime}(1), \beta_{2}^{\prime}(1)\right)\left(\alpha_{1}(0), \alpha_{2}(0)\right),
\end{aligned}
$$

a contradiction. Similarly if $\psi$ is no nincreasing we get same contradiction. Consequently, (2.4) holds. By definition of $\psi^{*}$ and Step 2, the second boundary condition is obvious. Consequently, $(u, v)$ satisfies (1.2).

Step 3: If $(u, v) \in C^{2}[0,1] \times C^{2}[0,1]$ is a solution of $(2.2)$ then $(-M,-M)\left(u^{\prime}(t), v^{\prime}(t)\right)(M, M)$.

$$
-M<-r_{1} \leq \alpha_{1}(1)-\beta_{1}(0)+\alpha_{2}(1)-\beta_{2}(0) \leq u^{\prime}\left(s_{0}\right)+v^{\prime}\left(s_{0}\right) \leq \beta_{1}(0)-\alpha_{1}(0)+\beta_{2}(0)-\alpha_{2}(0) \leq r_{1}<M .
$$

Now consider an interval $\left[t_{1}, t_{2}\right]$ or $\left[t_{2}, t_{1}\right]$ such that $u^{\prime}\left(t_{1}\right)+v^{\prime}\left(t_{1}\right)=r_{1}$ and $u^{\prime}\left(t_{2}\right)+v^{\prime}\left(t_{2}\right)=M$, with

$$
r_{1}=u^{\prime}\left(t_{1}\right)+v^{\prime}\left(t_{1}\right) \leq u^{\prime}(t)+v^{\prime}(t) \leq u^{\prime}\left(t_{2}\right)+v^{\prime}\left(t_{2}\right)=M, t \in\left(t_{1}, t_{2}\right)
$$

or

$$
r_{1}=u^{\prime}\left(t_{1}\right)+v^{\prime}\left(t_{1}\right) \leq u^{\prime}(t)+v^{\prime}(t) \leq u^{\prime}\left(t_{2}\right)+v^{\prime}\left(t_{2}\right)=M, t \in\left(t_{2}, t_{1}\right) .
$$

In the first situation we obtain form (1.2) that

$$
\begin{aligned}
& \int_{u^{\prime}\left(t_{1}\right)+v^{\prime}\left(t_{1}\right)}^{u^{\prime}\left(t_{2}\right)+v^{\prime}\left(t_{2}\right)} \frac{d y}{\xi(y)}=\int_{i_{1}}^{M} \frac{d y}{\xi(y)}>2 . \\
& \int_{u^{\prime}\left(t_{1}\right)+v^{\prime}\left(t_{1}\right)}^{u^{\prime}\left(t_{2}\right)+v^{\prime}\left(t_{2}\right)} \frac{d y}{\xi(y)}=\int_{t_{1}}^{t_{2}} \frac{u^{\prime \prime}(t)+v^{\prime \prime}(t)}{\xi\left(u^{\prime}(t)+v^{\prime}(t)\right)} d t=\int_{t_{1}}^{t_{2}} \frac{u^{\prime \prime}(t)}{\xi\left(u^{\prime}(t)+v^{\prime}(t)\right)} d t+\int_{t_{1}}^{t_{2}} \frac{v^{\prime \prime}(t)}{\xi\left(u^{\prime}(t)+v^{\prime}(t)\right)} d t \\
& =\int_{t_{1}}^{t_{2}} \frac{-F^{*}\left(t, u(t), v(t), u^{\prime}(t), v^{\prime}(t)\right)+\lambda u(t)}{\xi\left(u^{\prime}(t)+v^{\prime}(t)\right)} d t+\int_{t_{1}}^{t_{2}} \frac{-G^{*}\left(t, u(t), v(t), u^{\prime}(t), v^{\prime}(t)\right)+\lambda v(t)}{\xi\left(u^{\prime}(t)+v^{\prime}(t)\right)} d t \\
& =\int_{t_{1}}^{t_{2}} \frac{-f\left(t, u(t), v(t), u^{\prime}(t), v^{\prime}(t)\right)}{\xi\left(u^{\prime}(t)+v^{\prime}(t)\right)} d t+\int_{t_{1}}^{t_{2}} \frac{-g\left(t, u(t), v(t), u^{\prime}(t), v^{\prime}(t)\right)}{\xi\left(u^{\prime}(t)+v^{\prime}(t)\right)} d t \\
& \leq \int_{t_{1}}^{t_{2}} \frac{\left|f\left(t, u(t), v(t), u^{\prime}(t), v^{\prime}(t)\right)\right|}{\xi\left(u^{\prime}(t)+v^{\prime}(t)\right)} d t+\int_{t_{1}}^{t_{2}} \frac{\left|g\left(t, u(t), v(t), u^{\prime}(t), v^{\prime}(t)\right)\right|}{\xi\left(u^{\prime}(t)+v^{\prime}(t)\right)} d t \\
& \leq \int_{t_{1}}^{t_{2}} \frac{\xi\left(u^{\prime}(t)+v^{\prime}(t)\right)}{\xi\left(u^{\prime}(t)+v^{\prime}(t)\right)} d t+\int_{t_{1}}^{t_{2}} \frac{\xi\left(u^{\prime}(t)+v^{\prime}(t)\right)}{\xi\left(u^{\prime}(t)+v^{\prime}(t)\right)} d t \leq \int_{t_{1}}^{t_{2}} d t+\int_{t_{1}}^{t_{2}} d t \leq 2 .
\end{aligned}
$$

Using (1.6), Step 2 and $M \geq u^{\prime}(t)+v^{\prime}(t) \geq r_{1} \geq 0$, for all

Similarly in the second situation we get a contradiction. Hence $\left(u^{\prime}(t), v^{\prime}(t)\right) \prec(M, M)$. The proof of the other inequality is similar.

\section{Example}

Example 3.1: Consider the nonlinear coupled boundary value system (BVS for short) with nonlinear CBCs

$$
\left.\left.\begin{array}{r}
-u^{\prime \prime}(t)=\sin ^{2}(\pi t)-5\left(u^{\prime}(t)+v^{\prime}(t)\right)-(u(x)+1)^{2} \\
-(v(x)+1)^{2}, \quad t \in[0,1], \\
-v^{\prime \prime}(t)=\sin ^{2}(\pi t)-10\left(u^{\prime}(t)+2 v^{\prime}(t)\right)-(u(x)+2)^{4} \\
-(v(x)+1)^{4}, \quad t \in[0,1],
\end{array}\right\} \begin{array}{r}
\left(u(0) u^{\prime}(0)-v(0) v^{\prime}(0), u(0) u^{\prime}(1)-v(0) v^{\prime}(1)\right)=(0,0), \\
(u(0) v(0)+u(0) v(1), u(1) v(0)+u(0) v(1))=(0,0) .
\end{array}\right\}
$$

Let $\alpha_{1}(t)=-t^{2}-t, \alpha_{2}(t)=-t$ and $\beta_{1}(t)=t^{2}+t, \beta_{2}(t)=t$ are the coupled lower and upper solutions of the BVS (3.1)-(3.2). Consequently 


$$
\begin{array}{ll}
-\alpha_{1}^{\prime \prime}(t) \leq f\left(t, \alpha_{1}(t), \alpha_{2}(t), \alpha_{1}^{\prime}(t), \alpha_{2}^{\prime}(t)\right), & t \in[0,1], \\
-\alpha_{2}^{\prime \prime}(t) \leq g\left(t, \alpha_{1}(t), \alpha_{2}(t), \alpha_{1}^{\prime}(t), \alpha_{2}^{\prime}(t)\right), & t \in[0,1],
\end{array}
$$

and

$$
\begin{array}{ll}
-\beta_{1}^{\prime \prime}(t) \geq f\left(t, \alpha_{1}(t), \alpha_{2}(t), \alpha_{1}^{\prime}(t), \alpha_{2}^{\prime}(t)\right), & t \in[0,1], \\
-\beta_{1}^{\prime \prime}(t) \geq g\left(t, \beta_{1}(t), \beta_{2}(t), \beta_{1}^{\prime}(t), \beta_{2}^{\prime}(t)\right), & t \in[0,1] .
\end{array}
$$

Furthermore the coupled lower and upper solutions satisfies the system (2.1). And the functions

$$
\begin{gathered}
f(t, x, y, z, w)=\sin ^{2}(\pi t)-5(z+w)-(x+1)^{2}-(y+1)^{2} \\
g(t, x, y, z, w)=\sin ^{2}(\pi t)-10(z+2 w)-(x+2)^{4}-(y+1)^{4}
\end{gathered}
$$

satisfies the Nagumo condition (1.6) with $\xi(z+w)=-5(z+w)-1, z, w \in \mathbb{R} \quad$ and $\xi(z+w)=-10(z+w), z, w \in \mathbb{R}$ respectively. Hence by Theorem (2.2), BVS (3.1)-(3.2) has at least one solution $(u, v) \in \llbracket \alpha_{1}, \beta_{1} \rrbracket \times \llbracket \alpha_{2}, \beta_{2} \rrbracket$.

\section{References}

[1] R. P. Agarwal and D. O'Regan, A coupled system of boundary value problems. Appl. Anal. 69 (1998), no. 3-4, 381-385.

[2] N. A. Asif and R. A. Khan, Positive solutions to singular system with four-point coupled boundary conditions. J. Math. Anal. Appl. 386 (2012), no. 2, 848-861.

[3] Z. Bai and W. Ge, Existence of three positive solutions for some second-order boundary value problems. Comput. Math. Appl. 48 (2004), 699-707.

[4] X. Cheng and C. Zhong, Existence of positive solutions for a second-order ordinary differential system. J. Math. Anal. Appl. 312 (2005), no. 1, 14-23.

[5] D. Franco, J. J. Nieto, and D. O'Regan, Anti-periodic boundary value problem for nonlinear first order ordinary differential equations. Math. Inequal. Appl. 6 (2003), no. 3, 477-485.
[6] J. Henderson and R. Luca, Positive solutions for a system of second-order multi-point boundary value problems. Appl. Math. Comput. 218 (2012), no. 10, 6083-6094.

[7] J. Henderson and H.B. Thompson, Existence of multiple solutions for second-order boundary value problems. J. Differential. Eqns. 166 (2000), 443-454.

[8] T. Jankowski, Ordinary differential equations with nonlinear boundary conditions of antiperiodic type. Comput. Math. Appl. 47 (2004), no. 8-9, 1419-1428.

[9] J. Jiang, L. Liu, and Y. Wu, Symmetric positive solutions to singular system with multi-point coupled boundary conditions. Appl. Math. Comput. 220 (2013), 536-548.

[10] V. Lakshmikantham, Periodic boundary value problems of first and second order differential equations. J. Appl. Math. Simulation 2 (1989), no. 3, 131-138.

[11] J. J. Nieto, Periodic boundary value problems for first-order impulsive ordinary differential equations. Nonlinear Anal. 51 (2002), no. 7, 1223-1232.

[12] H. Qu, The symmetric positive solutions of three-point boundary value problems for nonlinear second-order differential equations. Bulletin of the institute of mathematics academia Sinica. 7 (2012), no. 3, 405-416.

[13] K. Wang, A new existence result for nonlinear first-order antiperiodic boundary value problems. Appl. Math. Letters. 21(2008), 1149-1154.

[14] J. Wu, Theory and applications of partial functionaldifferential equations. Applied Mathematical Sciences, 119. Springer-Verlag, New York, 1996.

[15] W. Zhang and M. Fan, Periodicity in a generalized ecological competition system governed by impulsive differential equations with delays. Math. Comput. Modelling 39 (2004), no. 4-5, 479-493.

[16] Y. Zhang, Positive solutions of singular sublinear EmbdenFowler boundary value problems. J. Math. Anal. Appl. 185 (1994), 215-222.

[17] Y. Zhang, The existence of solutions to nonlinear second-order periodic boundary value problems. Nonlinear Anal. 76 (2013), $140-152$. 